Supporting Information for

\title{
Identification of a novel inhibitor of catabolite control protein A from Staphylococcus aureus
}

Qi Huang, ${ }^{\ddagger a}$ Zhemin Zhang, ${ }^{\ddagger a}$ Huinan Li, ${ }^{\ddagger a}$ Yu Guo, ${ }^{\text {a } X i a n g w e n ~ L i a o, ~}{ }^{\text {a }}$ Hongyan Li, ${ }^{\mathrm{b}}$ Huihao Zhou $^{\mathrm{c}}$ and Wei Xia ${ }^{{ }^{*} \mathrm{a}}$

a. MOE Key Laboratory of Bioinorganic and Synthetic Chemistry, School of Chemistry, Sun Yat-sen University, No. 135, Xingang Xi Road, Guangzhou, China, 510275.

Email: xiawei5@mail.sysu.edu.cn

b. Department of Chemistry, The University of Hong Kong, Pokfulam Road, Hong Kong, China.

c. Research Center for Drug Discovery, School of Pharmaceutical Sciences, Sun Yat-sen University, 132 Outer Ring East Road, Guangzhou, 510006, China 


\section{Table of content}

Fig. S1. Fluorescent-based screening of $\mathrm{SaCcpA}$ binding to the small molecule compounds S3

Fig. S2. Validation of lead compounds binding to $S a C c p A$ S4

Fig. S3. Electrophoretic mobility shift assay of $S a C c p A$ binding to $p c k A$ probe in the presence of gradient amounts of ABT S5

Fig. S4. $\Delta T m$ of wild-type $S a C c p A$ and different mutants incubated with TDP. S6

Fig. S5. $\Delta T m$ of $S a C c p A$ incubated TDP and six derivatives. ..S7

Fig. S6. Growth curves of wild-type $S$. aureus and S. aureus $\Delta c c p A$ mutant incubated with $150 \mu \mathrm{M}$ TDP and six derivatives. S8

Fig. S7. Growth curves of wild-type $S$. aureus and $S$. aureus $\Delta c c p A$ mutant in the presence of gradient concentrations of HMS S9

Fig. S8. Quantitative transcript analysis of $p c k A$, hla and $c c p A$ genes in wild-type $S$. aureus after incubation with 5 $\mu \mathrm{M}$ HMS. S10

Fig. S9. Quantitative transcript analysis of $p c k A$ and $c c p A$ genes in wild-type Bacillus subtilis after incubation with $20 \mu \mathrm{M}$ HMS for $3 \mathrm{~h}$. ....

Fig. S10. Bactericidal activity of murine macrophage $\mathrm{J} 774$ cell toward $S$. aureus or $\Delta c c p A$ mutant treated with or without HMS compound. $\mathrm{S} 12$

Fig. S11. Heat plots of checkerboard assays for HMS in combination with different antibiotics against $S$. aureus Newman. S13

Fig. S12. Cytotoxity of HMS to normal human hepatic cell line L02 by MTT assay..... ..S14

Fig. S13. Heat plots of checkerboard assays for HMS in combination with gentamicin against MRSA..... S15

Fig. S14. Uptake of propidium iodide by S. aureus Newman treated with indicated concentration of bithionol or HMS. S16

Fig. S15. Heat plots of checkerboard assays for HMS in combination with different aminoglycoside antibiotics against $S$. aureus $\triangle c c p A$ mutant. S17

Tab. S1. Strains, plasmids and primers S18 
A

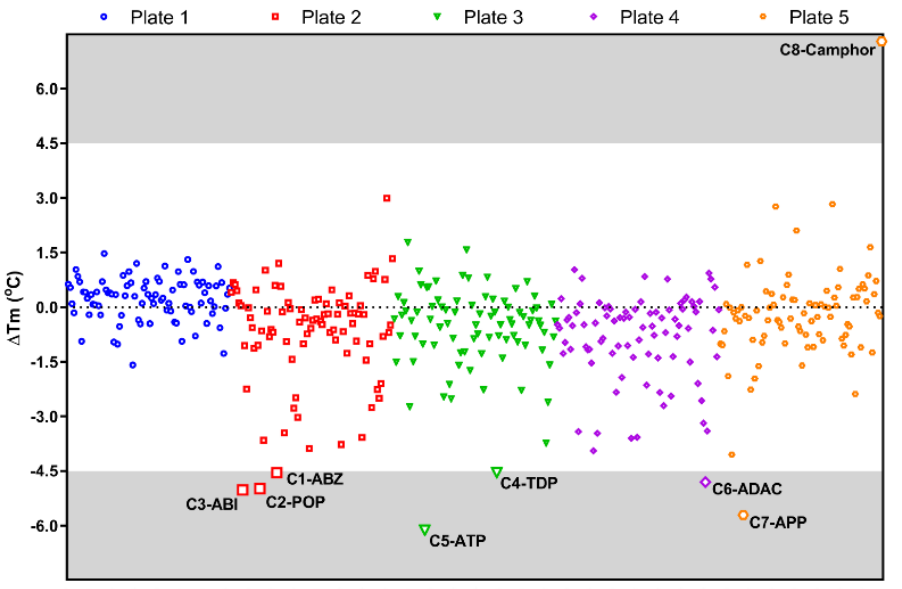

B

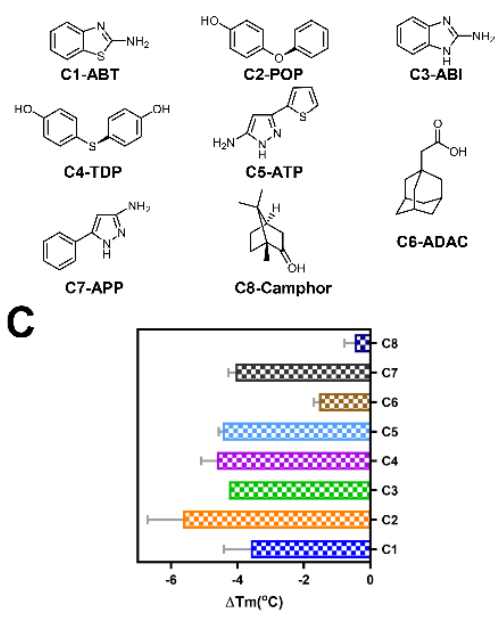

Fig. S1. (A) Fluorescent-based protein thermal shift assays of $\mathrm{SaCcpA}$ binding to the small molecule compounds. The $\Delta T_{m}$ values of $S a \mathrm{CcpA}$ incubated with each compound are shown. Compounds with $|\Delta T m|>4.5^{\circ} \mathrm{C}$ were labeled. (B) Structures of the identified hit compounds in the initial screening. C1-ABT: 2-aminobenzothiazole; C2-POP: 4-phenoxyphenol; C3-ABI: 2aminobenzimidazole; C4-TDP: 4,4'-thiodiphenol; C5-ATP: 5-amino-3-(2-thienyl)pyrazole; C6ADAC: 1-adamantaneacetic acid; C7-APP: 3-amino-5-phenylpyrazole; C8-Camphor: 2,3bornanedione. (C) $\Delta T_{m}$ of $S a \mathrm{CcpA}$ incubated with $\mathrm{C} 1-\mathrm{C} 8$ compounds in the rescreening process. All experiments were performed in triplicate and results are shown as mean $\pm \mathrm{sd}$. 
A

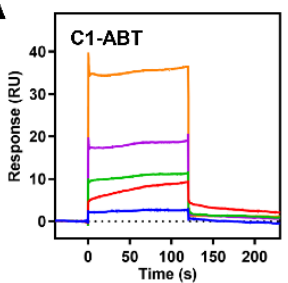

$-6.25 \mu \mathrm{M}$

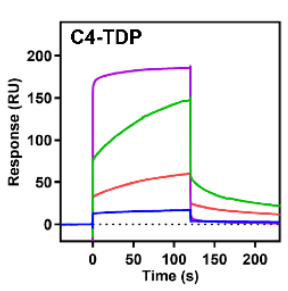

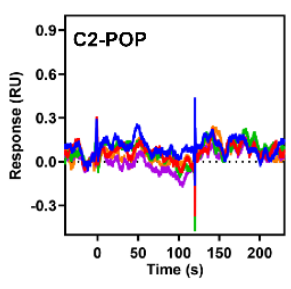

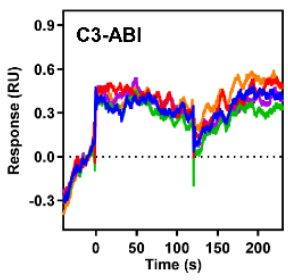

$-100 \mu \mathrm{M}$
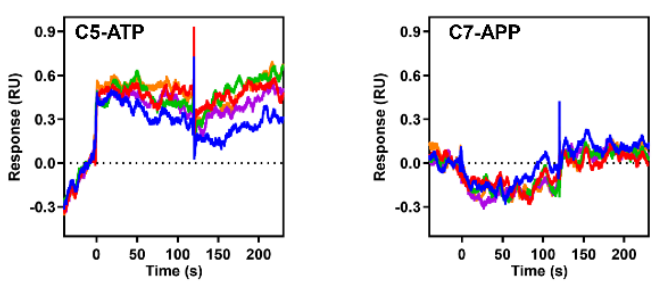

B
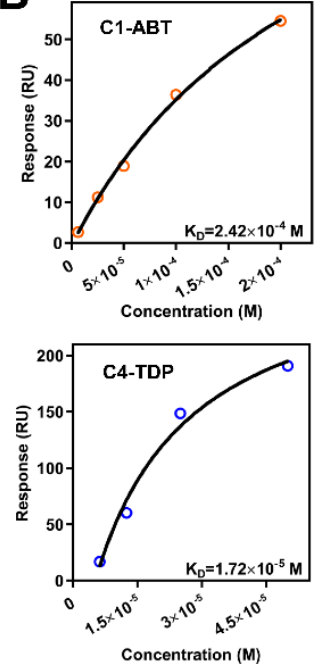

Fig. S2. (A) Sensorgrams of $\mathrm{SaCcpA}$ binding to gradient concentrations of 8 hit compounds. Only $\mathrm{C} 1$ and $\mathrm{C} 4$ exhibited detectable binding to $\mathrm{SaCcpA}$. (B) Equilibrium data analysis of $\mathrm{C} 1$ and $\mathrm{C} 4$ binding to $S a C c p A$. The plots of steady state response units (RU) against molecule concentrations were fitted to a single-site interaction model. 


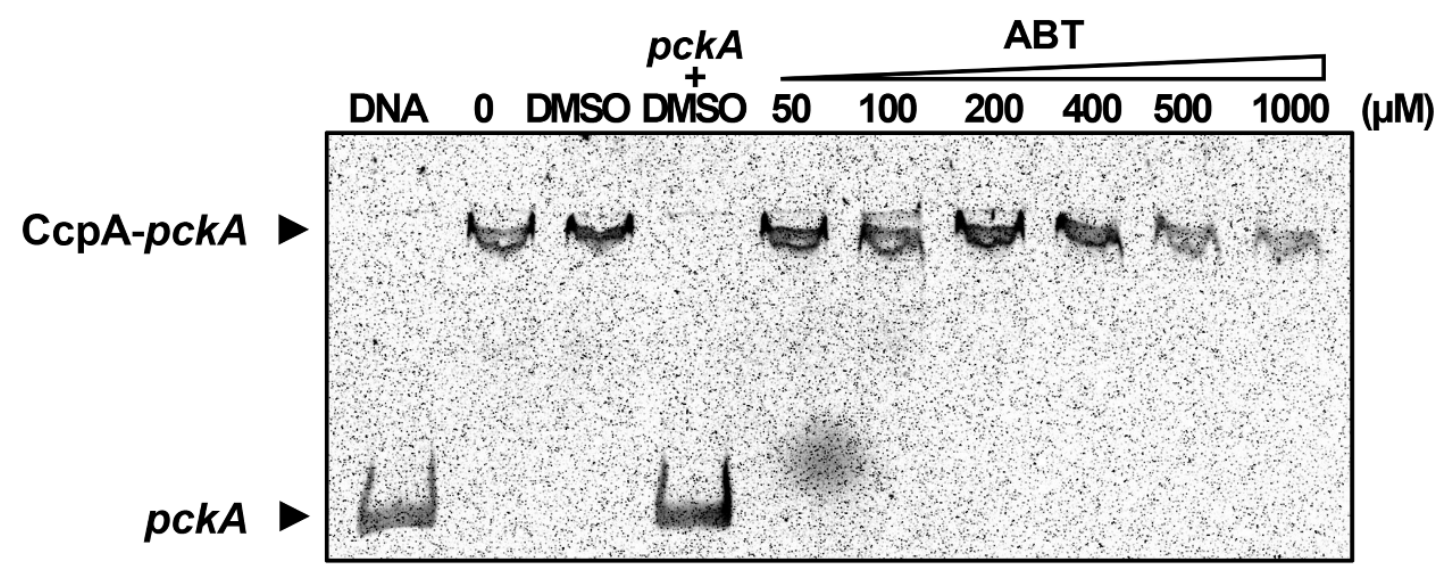

Fig. S3. Electrophoretic mobility shift assay (EMSA) of $S a C c p A$ binding to $p c k A$ probe in the presence of gradient amounts of 2-aminobenzothiazole (ABT). 


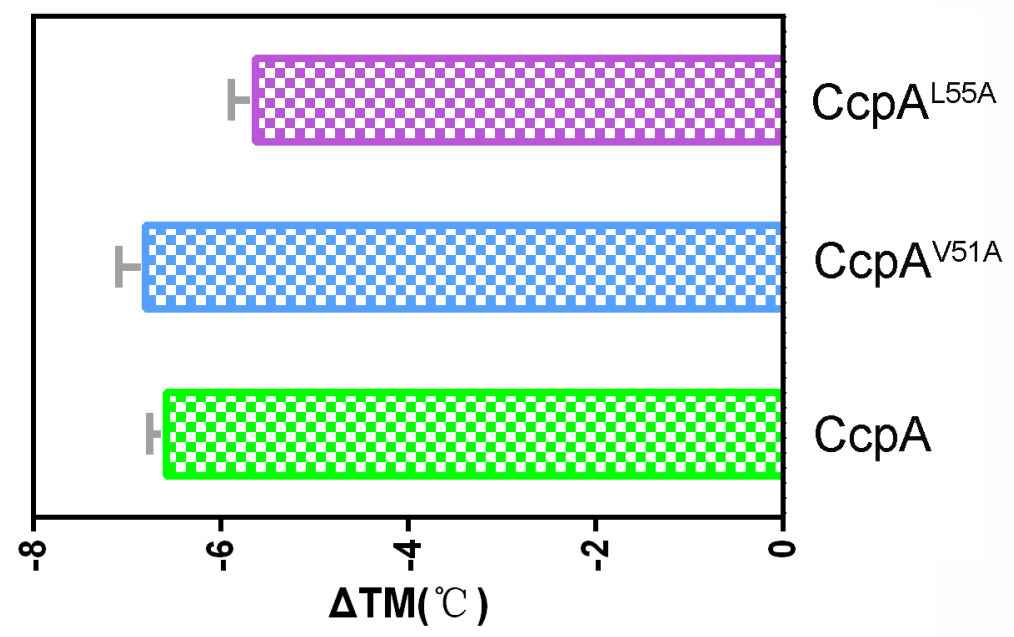

Fig S4. $\Delta T m$ of wild-type $S a C c p A$ and different mutants incubated with 4,4'-thiodiphenol (TDP). 


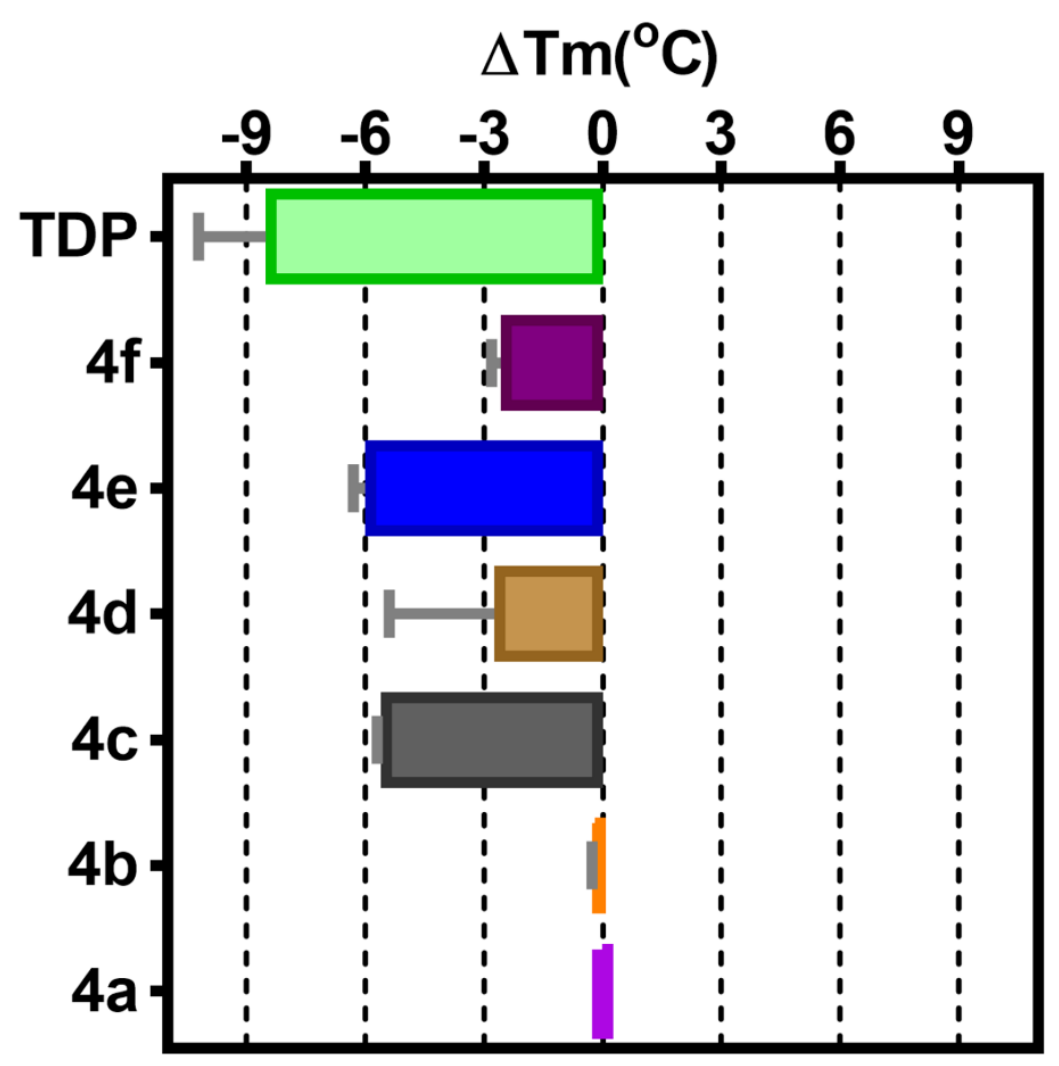

Fig S5. $\Delta T m$ of $S a C c p A$ incubated with 4,4'-thiodiphenol (TDP) and six derivatives. 4a: diphenyl sulfide; 4b: 4,4'-thiodianiline; 4c: 2,2',4,4'-tetrahydroxydiphenyl sulfide; 4d: 5,5'-hiodisalicylic acid; 4e: bis(4-hydroxy-3-methylphenyl) sulfide; 4f: 4,4'-dihydroxyazobenzene. All experiments were performed in triplicate and results are shown as mean $\pm \mathrm{sd}$. 

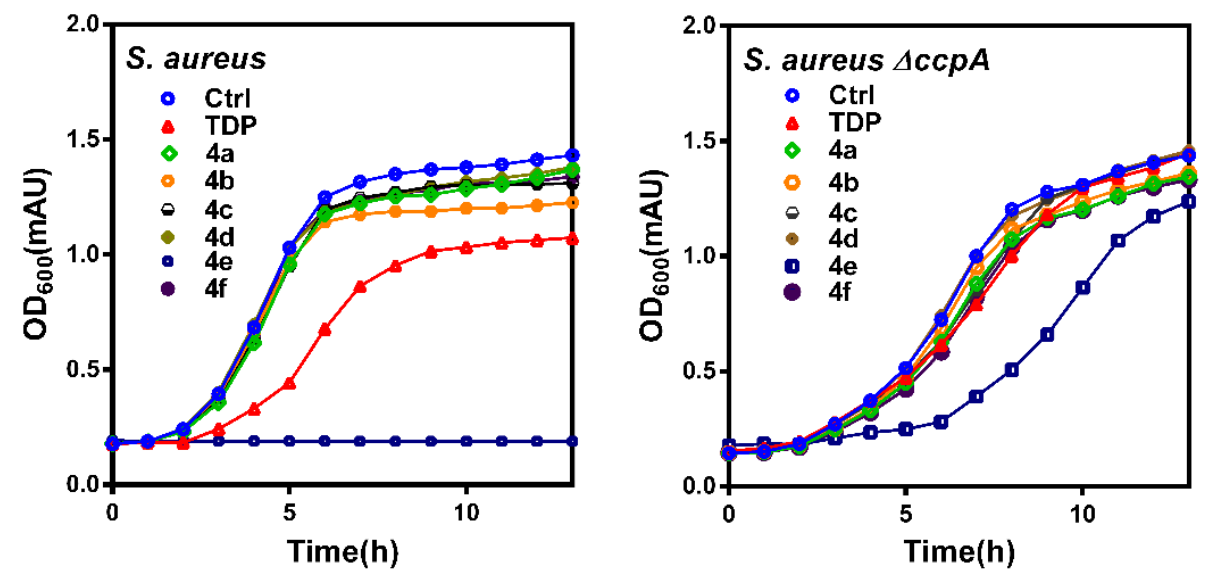

Fig S6. Growth curves of wild-type $S$. aureus (left) and $S$. aureus $\triangle$ ccpA mutant (right) incubated with $150 \mu \mathrm{M} 4,4^{\prime}$-thiodiphenol (TDP) and six derivatives. $0.1 \%$ DMSO was used as a negative control. 

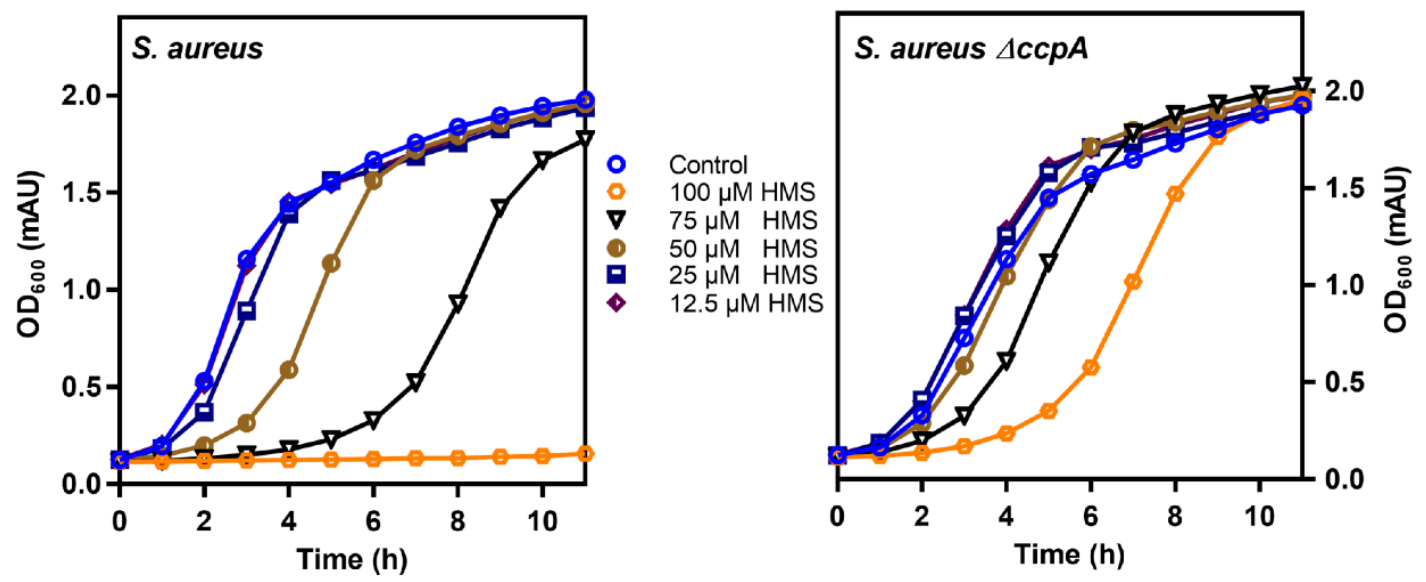

Fig S7. Growth curves of wild-type $S$. aureus (left) and $S$. aureus $\Delta c c p A$ mutant (right) in the presence of gradient concentrations of bis(4-hydroxy-3-methylphenyl) sulfide (HMS). 


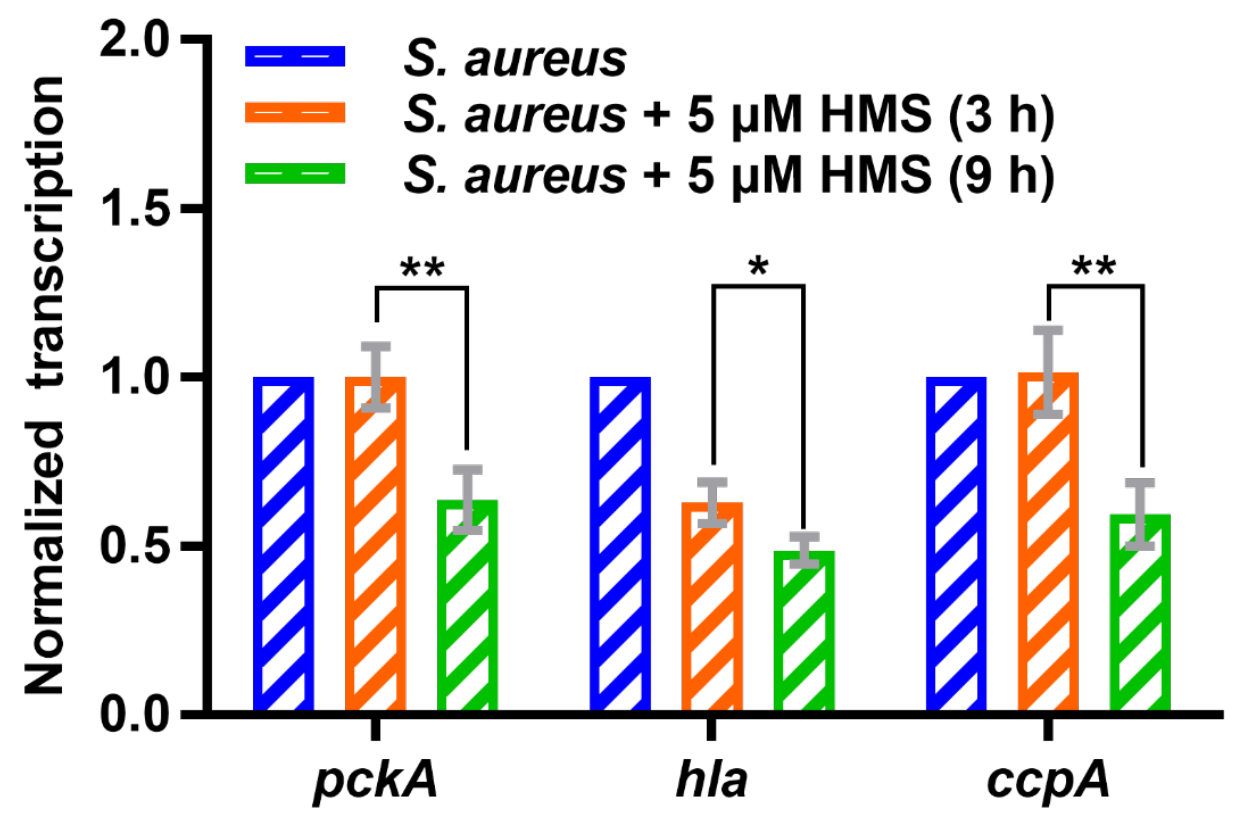

Fig S8. Quantitative transcript analysis of $p c k A$, hla and $c c p A$ genes in wild-type $S$. aureus after incubation with $5 \mu \mathrm{M}$ bis(4-hydroxy-3-methylphenyl) sulfide (HMS) for $3 \mathrm{~h}$ and $9 \mathrm{~h}$. The mean value of transcription level in wild type $S$. aureus control groups was set as 1 . The transcription levels in other groups were normalized to that of control group. All experiments were performed in triplicate and results are shown as mean $\pm \mathrm{sd}$. The statistical difference is determined by two-tailed Student's t-test. * indicates $\mathrm{p}<0.05$; ** indicates $\mathrm{p}<0.01$. 
$\infty$ B. subtilis

B. subtilis $+20 \mu \mathrm{M} \mathrm{HMS}$

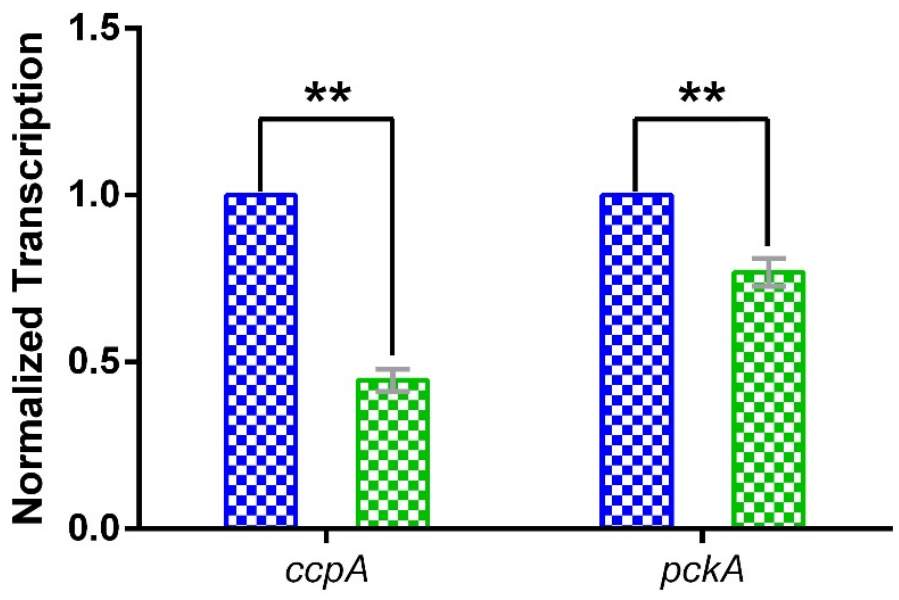

Fig S9. Quantitative transcript analysis of $p c k A$ and $c c p A$ genes in wild-type Bacillus subtilis after incubation with $20 \mu \mathrm{M}$ bis(4-hydroxy-3-methylphenyl) sulfide (HMS) for $3 \mathrm{~h}$. The mean value of transcription level in wild-type Bacillus subtilis control groups was set as 1. The transcription levels in other groups were normalized to that of control group. All experiments were performed in triplicate and results are shown as mean $\pm \mathrm{sd}$. The statistical difference is determined by two-tailed Student's t-test. * indicates $\mathrm{p}<0.05 ; * *$ indicates $\mathrm{p}<0.01$. 


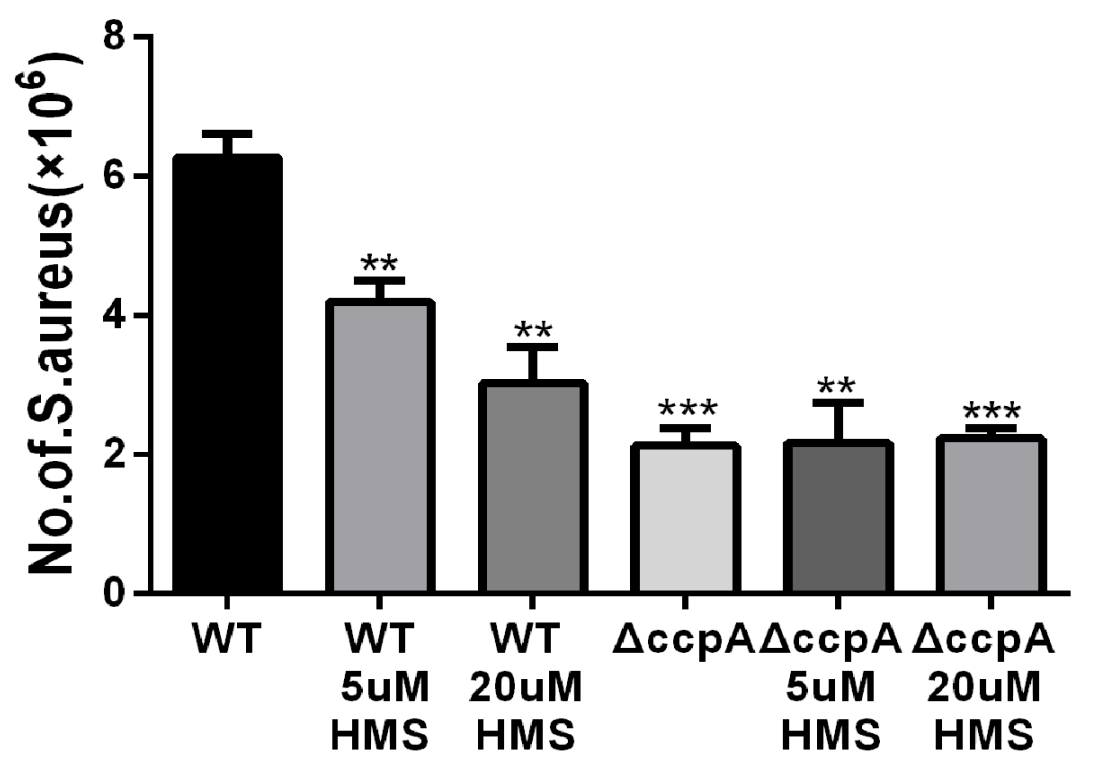

Fig S10. Bactericidal activity of murine macrophage $\mathrm{J} 774$ cell toward $S$. aureus or $\Delta c c p A$ mutant treated with or without HMS compound. The survival number of bacteria after macrophage phagocytosis are shown. All experiments were performed in triplicate and results are shown as mean \pm sd. The statistical difference is determined by two-tailed Student's t-test. $*$ indicates $\mathrm{p}<0.05$; ** indicates $\mathrm{p}<0.01$. 

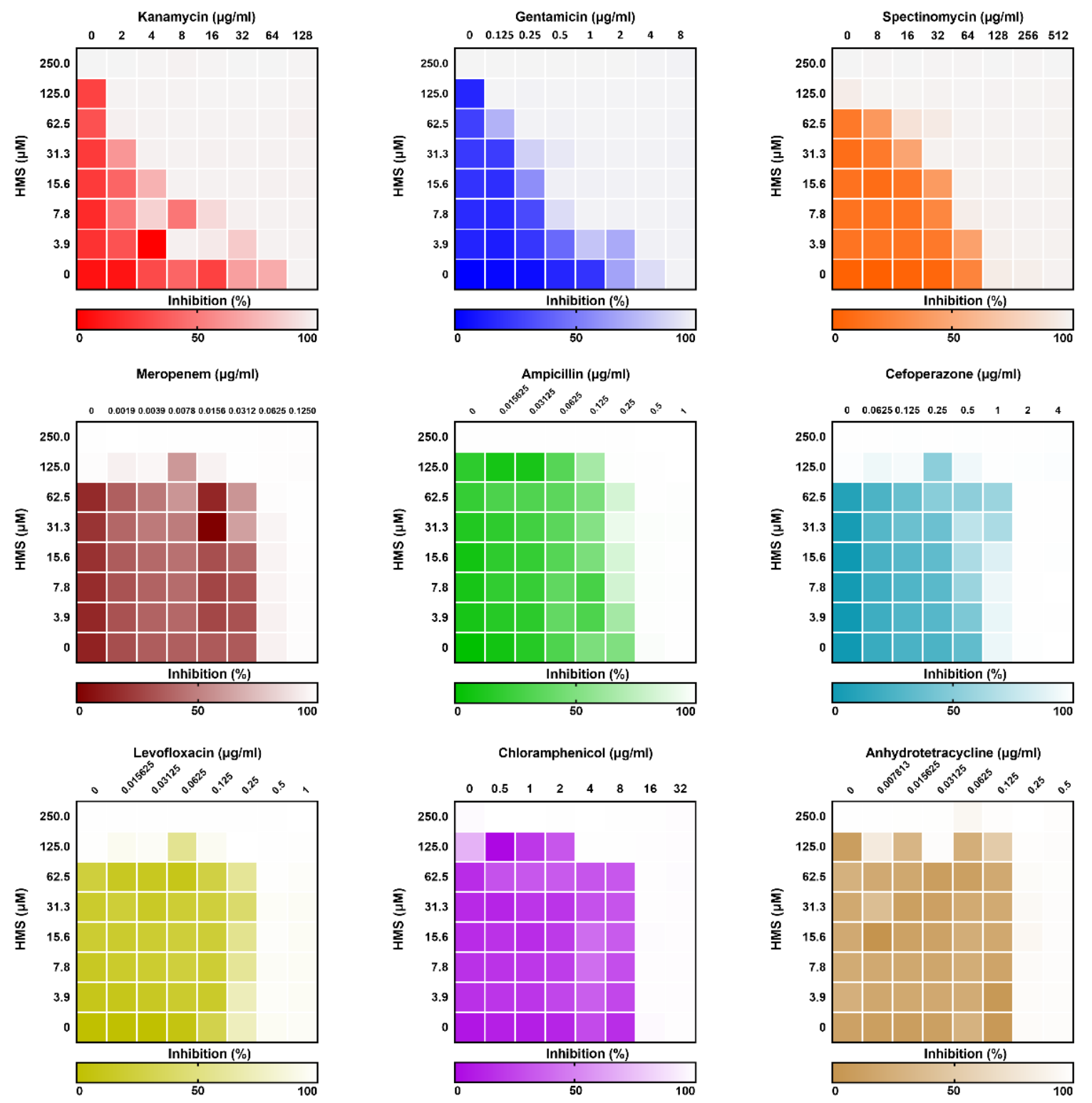

Fig. S11. Heat plots of checkerboard assays for HMS in combination with different antibiotics against $S$. aureus Newman. 


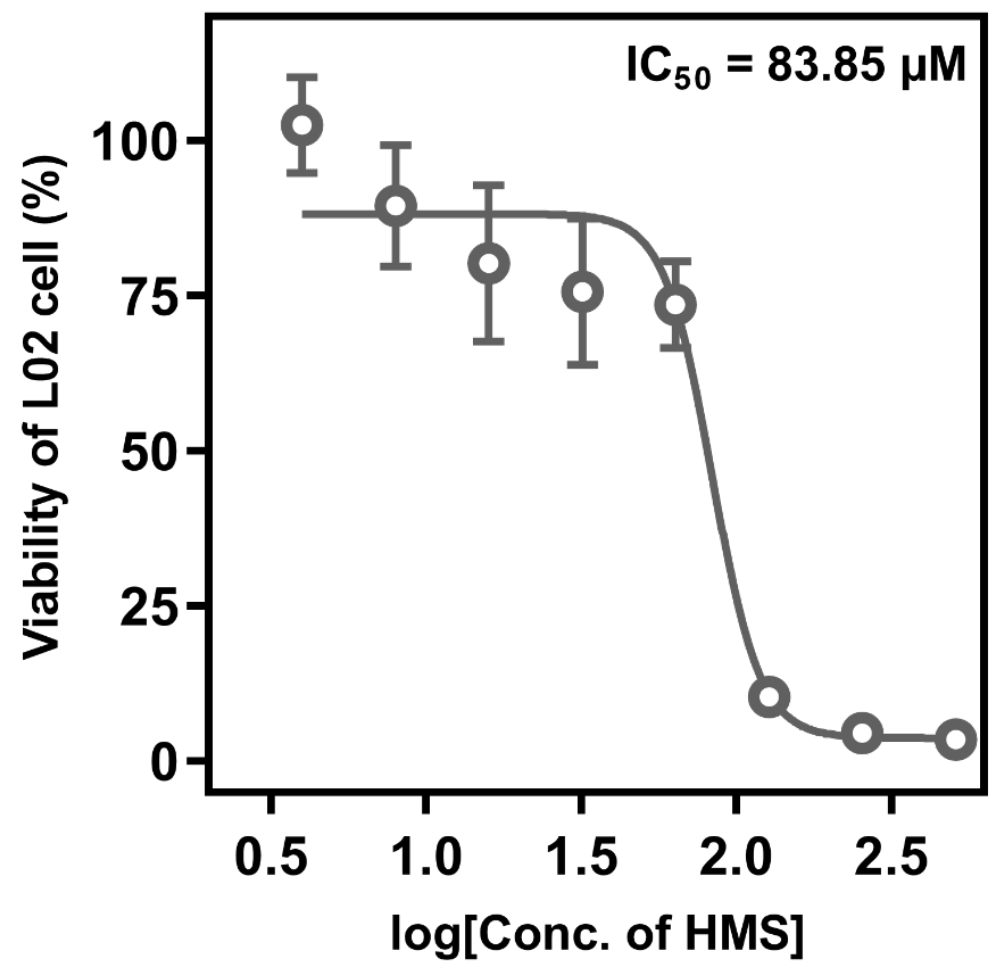

Fig. S12. Cytotoxity of HMS to normal human hepatic cell line L02 by MTT assay. 


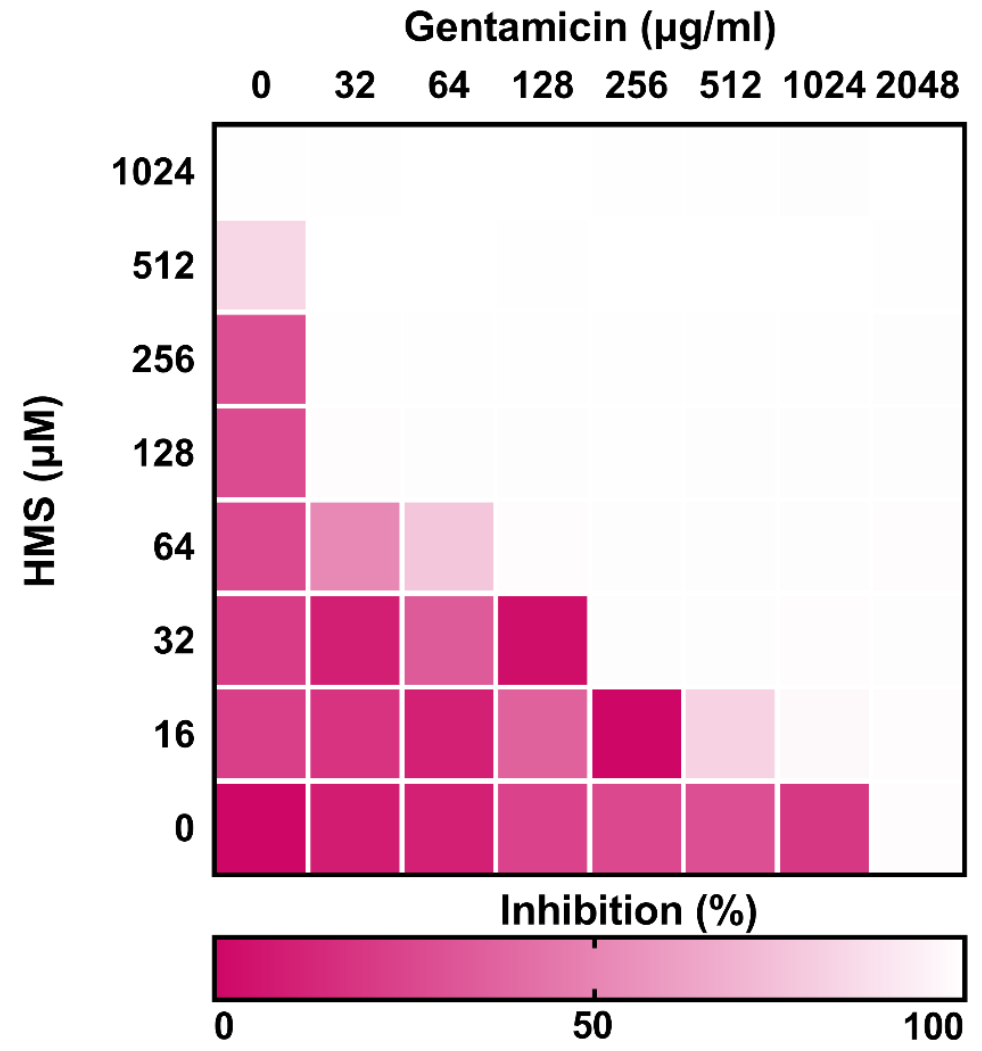

Fig. S13. Heat plots of checkerboard assays for HMS in combination with gentamicin against MRSA. 


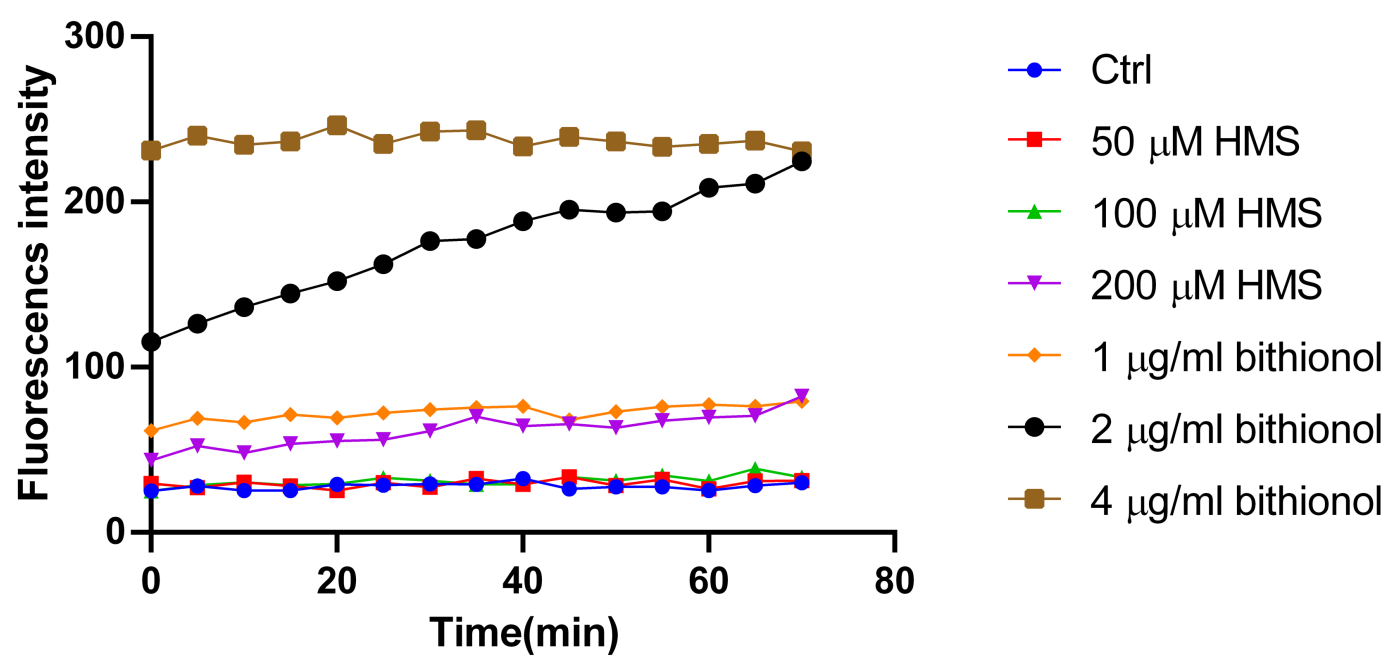

Fig. S14. Uptake of propidium iodide (PI) by $S$. aureus Newman treated with indicated concentration of bithionol or bis(4-hydroxy-3-methylphenyl) sulfide (HMS). All experiments were performed in triplicates. Results are shown as means, error bars are not shown for clarity. 

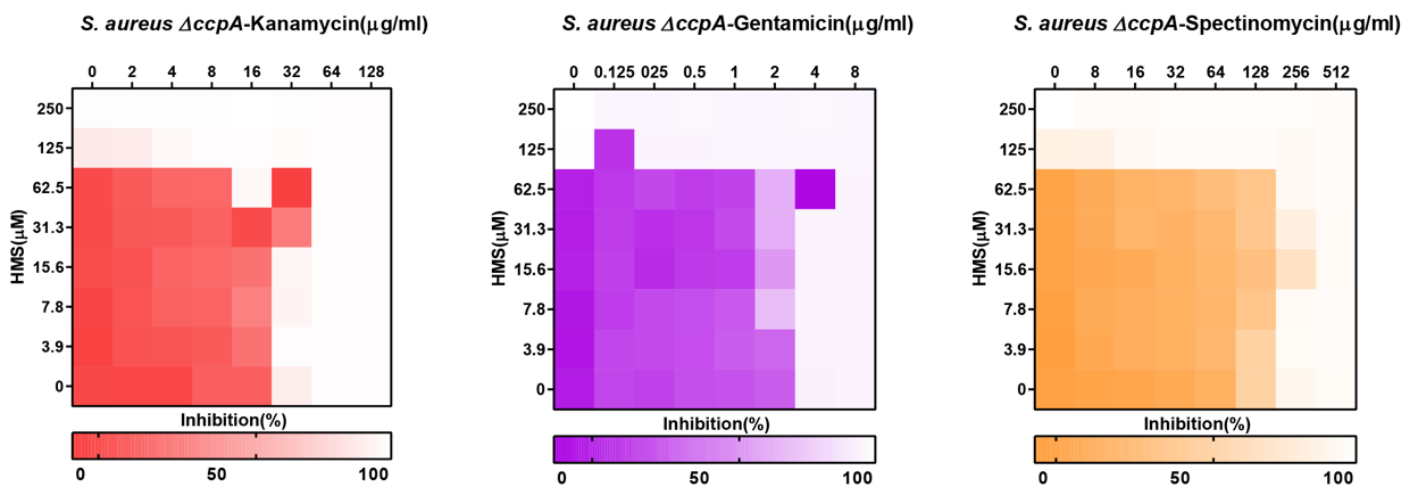

Fig. S15. Heat plots of checkerboard assays for HMS in combination with different aminoglycoside antibiotics against $S$. aureus $\triangle c c p A$ mutant. 
Table S1. Strains, plasmids and primers

\begin{tabular}{|c|c|c|}
\hline $\begin{array}{c}\text { Strains, plasmids or } \\
\text { primers }\end{array}$ & \multicolumn{2}{|c|}{ Application } \\
\hline \multicolumn{3}{|l|}{ E. coli strains } \\
\hline XL1-Blue & \multicolumn{2}{|l|}{ Plasmid maintenance } \\
\hline $\mathrm{BL} 21\left(\mathrm{DE}_{3}\right)$ & \multicolumn{2}{|l|}{ Protein expression } \\
\hline \multicolumn{3}{|c|}{ Staphylococcus aureus strains } \\
\hline RN4220 & \multicolumn{2}{|l|}{ Modification plus strain } \\
\hline Newman & \multicolumn{2}{|l|}{ Wild-type $S$. aureus Newman. } \\
\hline Newman $\triangle c c p A$ & \multicolumn{2}{|l|}{$c c p A$ gene-knockout strain. } \\
\hline \multicolumn{3}{|l|}{ Plasmids } \\
\hline pET47b-cсpA & \multicolumn{2}{|l|}{ CcpA protein expression } \\
\hline \multicolumn{3}{|l|}{ Primers for EMSA } \\
\hline pckA & $\begin{array}{l}\text { GCTGGGTTTCATTGGGTCCATGTCC } \\
\text { CT }\end{array}$ & $\begin{array}{l}\text { GCTGGGTTTCATTGGGTCCATGTCC } \\
\text { CT }\end{array}$ \\
\hline \multicolumn{3}{|l|}{ Primers for $q R T-P C R$} \\
\hline 16s RNA & CCATAAAGTTGTTCTCAGTT & CATGTCGATCTACGATTACT \\
\hline pckA & CGTTGGTGAAAAAGGCGATGT & GCATAGCAGCCACCTTCGAT \\
\hline hla & AACCCGGTATATGGCAATCAACT & CTGCTGCTTTCATAGAGCCATTT \\
\hline cсpA & AAAGGCAATTTGCCAGATGC & AATTGCTTCTTCGTCGCTGATAC \\
\hline
\end{tabular}

